\title{
Ações realizadas pelo acompanhante durante os cuidados imediatos com o recém- nascido em maternidades públicas
}

Actions performed by the companion during the immediate care of the newborn in public maternity hospitals

Acciones realizadas por el acompañante durante los cuidados inmediatos al recién nacido en maternidades públicas

\section{Mayara Carminatti SabinoI, Roberta Costa ${ }^{\text {II }}$, Manuela Beatriz Velho ${ }^{I I I}$, Odaléa Maria Brüggemann $^{\mathrm{IV}}$, Carolina Frescura Jungesv ${ }^{\text {, Iris Elizabete Messa Gomes }}{ }^{\mathrm{vI}}$}

Resumo: Objetivo: descrever as ações realizadas pelo acompanhante da mulher durante os cuidados imediatos com o recém-nascido. Método: estudo quantitativo descritivo realizado em três maternidades públicas da Grande Florianópolis, Santa Catarina, Brasil, com 1.075 entrevistados entre março de 2015 e maio de 2016; e aplicou-se análise estatística descritiva. Resultados: nas ações realizadas destacaram-se: conversar com o recém-nascido $(94,8 \%)$; acariciar (93,0\%); acalmar (78,3\%); pegar no colo (81,4\%); auxiliar na primeira mamada (67,6\%); e, conduzi-lo até a mãe $(58,4 \%)$. Foram menos frequentes: dar o primeiro banho (7,9\%); cortar o cordão umbilical $(20,4 \%)$; e, colocar a fralda $(26,7 \%)$. Conclusão: a participação do acompanhante nos cuidados com o recém-nascido demonstra ações de caráter sentimental, afetivo e de cuidado, bem como facilita o estabelecimento dos vínculos familiares. Conhecer as ações realizadas pelo acompanhante contribui para a prática assistencial, com a possibilidade de ampliar sua participação e proporcionar um maior significado para a mulher e à família.

Descritores: Acompanhantes formais em exames físicos; Apoio social; Enfermagem obstétrica; Parto humanizado; Recém-nascido

\footnotetext{
I Enfermeira. Universidade Federal de Santa Catarina, Florianópolis, SC, Brasil. E-mail: maynatti@msn.com ORCID: 0000-0001-5355-8233

II Enfermeira. Doutora. Professor Adjunto, Departamento de Enfermagem, Universidade Federal de Santa Catarina, Florianópolis, SC, Brasil. Email: roberta.costa@ufsc.br ORCID: 0000-0001-6816-2047

III Enfermeira. Doutora. Professor Adjunto, Departamento de Enfermagem, Universidade Federal de Santa Catarina, Florianópolis, SC, Brasil. E-mail: manuela.velho@ufsc.com ORCID 0000-0001-6660-6978

IV Enfermeira. Doutora. Professor Associado, Departamento de Enfermagem, Universidade Federal de Santa Catarina, Florianópolis, SC, Brasil. E-mail: odalea.ufsc@gmail.com ORCID: 0000-0003-1210-6040

V Enfermeira, Doutora. Hospital Universitário Polydoro Ernani de São Thiago, Universidade Federal de Santa Catarina, Florianópolis, SC, Brasil. E-mail: c.f.junges@ufsc.br ORCID: 0000-0001-7337-9646

VI Enfermeira, Mestre. Departamento de Enfermagem, Universidade Federal de Santa Catarina, Florianópolis, SC, Brasil. E-mail: irismessagomes@hotmail.com ORCID: 0000-0002-0496-5892
} 
Ações realizadas pelo acompanhante durante os cuidados imediatos com o recém-nascido... $\mid 2$

\begin{abstract}
Objective: to describe the actions performed by the woman's companion during immediate care of the newborn. Method: a descriptive quantitative study carried out in three public maternity hospitals in Greater Florianópolis, Santa Catarina, Brazil, with 1,075 respondents between March 2015 and May 2016; and descriptive statistical analysis was applied. Results: in the actions carried out, the following stood out: talking to the newborn (94.8\%); petting (93.0\%); calming down (78.3\%); picking up (81.4\%); assisting in first feeding $(67.6 \%)$; and leading him to the mother (58.4\%). The following were less frequent: giving the first bath (7.9\%); cutting the umbilical cord (20.4\%); and putting the diaper on (26.7\%). Conclusion: the companion's participation in caring for the newborn shows actions of a sentimental, affective and caring nature, as well as facilitating the establishment of family bonds. Knowing the actions taken by the companion contributes to the care practice, with the possibility of expanding their participation and providing greater meaning for the woman and the family.
\end{abstract}

Descriptors: Medical chaperones; Social support; Obstetric nursing; Humanized delivery; Infant, Newborn

Resumen: Objetivo: describir las acciones realizadas por el acompañante de la mujer durante los cuidados inmediatos del recién nacido. Método: estudio descriptivo cuantitativo realizado en tres maternidades públicas de la Gran Florianópolis, Santa Catarina, Brasil, con 1.075 encuestadas entre marzo de 2015 y mayo de 2016, por medio del análisis estadístico descriptivo. Resultados: se destacaron las siguientes acciones: hablar con el recién nacido (94,8\%); acariciarlo (93,0\%); calmar al recién nacido (78,3\%); alzarlo (81,4\%); ayudarlo en su primera alimentación (67,6\%); y llevarlo a su madre (58,4\%). Fueron menos frecuentes: darle el primer baño (7 ,9\%); cortar el cordón umbilical (20,4\%); y ponerle el pañal (26,7\%). Conclusión: la participación del acompañante en el cuidado del recién nacido demuestra acciones de carácter sentimental, afectivo y solidario, además de facilitar el establecimiento de lazos familiares. Conocer las acciones realizadas por el acompañante contribuye a la práctica del cuidado, con posibilidad de ampliar su participación y otorgar un mayor significado a la mujer y la familia.

Descriptores: Chaperones médicos; Apoyo social; Enfermería obstétrica; Trabajo de parto; Parto humanizado; Recién nacido

\title{
Introdução
}

A presença do acompanhante durante o processo de parturição é indicada por diretrizes nacionais e internacionais, que apontam esta como uma prática importante de apoio e suporte no cuidado à mulher. ${ }^{1-2}$ Considerada um marcador de segurança e qualidade na atenção ao parto, a presença do acompanhante também assegura uma aproximação com o recém-nascido, desde o momento do nascimento. ${ }^{2-3}$

No Brasil, a garantia da presença de um acompanhante de escolha da mulher, durante o trabalho de parto, parto e pós-parto imediato, está assegurada desde 2005, pela Lei n. 11.108, mais conhecida como a "Lei do acompanhante". Entretanto, na realidade nem sempre este 
direito é cumprido, o que foi evidenciado em estudo longitudinal que analisou dados da região sul do Brasil, nele $51,7 \%$ das parturientes tiveram a presença de acompanhante durante o trabalho de parto e, somente $39,4 \%$, no momento do parto. ${ }^{5}$

Como forma de contribuir positivamente para a experiência do nascimento, a presença de acompanhante pode se desenvolver com a participação passiva ou ativa deste. Passivamente, ficará como espectador, sem interferir nos acontecimentos, estando ao lado da mulher, numa atitude que demonstra empatia e afetividade. Nessa perspectiva, compartilhar esses acontecimentos se caracteriza como apoio e envolvimento contínuo, integrado ao processo do nascimento. ${ }^{6}$

Já na participação ativa, o acompanhante realiza atividades que fortaleçam a mulher, que a confortem e estimulem, seja de forma física ou emocional, desenvolvendo ações que a auxiliem na movimentação, na mudança de posição, na realização de massagem, assim como dar atenção, demonstrar carinho e dizer palavras de apoio e incentivo durante o trabalho de parto. Essa permanência propicia a vivência de momentos únicos que influenciam no fortalecimento das relações familiares e geram satisfação. ${ }^{6-7}$

A atuação do enfermeiro, estimulando a presença e participação do acompanhante durante todo o processo de parto e nascimento, é fundamental, sendo que este profissional fornece informações valiosas acerca das ações que podem ser desenvolvidas por aquele. ${ }^{8}$ Nesse contexto, identificá-las faz parte de estudos que pretendem compreender o papel e a participação do acompanhante, para que, assim, possam ser potencializados. Evidências listam os benefícios da presença de um acompanhante de escolha no processo de parto e nascimento, que, por meio das ações de apoio físico e emocional, promovem um maior grau de confiança nas mulheres, inibem mecanismos de dor, geram segurança e conforto..$^{6-7}$

No entanto, são escassas as produções nacionais e internacionais relativas à participação e os benefícios do acompanhante nos cuidados imediatos ao recém-nascido, aqueles prestados nas primeiras horas de vida. ${ }^{8-11}$ É possível encontrar estudos que citam suas ações nos cuidados 
Ações realizadas pelo acompanhante durante os cuidados imediatos com o recém-nascido... $\mid 4$

com o recém-nascido, mesmo que de forma secundária, ou guias que recomendam quais devam ser elas e, neste esteio, conhecer a atuação realizada pelo acompanhante se torna o escopo da presente pesquisa..$^{8,11-12}$

Acredita-se que conhecer e apresentar a realidade da atuação do acompanhante durante os cuidados imediatos com o recém-nascido pode repercutir de forma positiva no planejamento e acolhimento daquele, fazendo com que se sinta parte do processo de nascimento, elemento essencial para gerar vínculo, promover apoio e incentivar o aleitamento materno. Considerando a problemática, elaborou-se a seguinte questão de pesquisa: Quais são as ações desenvolvidas pelo acompanhante da mulher durante os cuidados imediatos ao recém-nascido? E, como objetivo, descrever as ações realizadas pelo acompanhante da mulher durante os cuidados imediatos com o recém-nascido.

\section{Método}

Pesquisa quantitativa de caráter descritivo que fez parte do macroprojeto “A participação do acompanhante de escolha da mulher no pré-natal, trabalho de parto e parto no sistema de saúde pública e suplementar”. A pesquisa obteve apoio financeiro do Conselho Nacional de Desenvolvimento Científico e Tecnológico (CNPq), processo número 473810/2013-1.

Os dados foram coletados no Alojamento Conjunto de três maternidades públicas da Grande Florianópolis, estado de Santa Catarina (SC), Brasil. A escolha desses cenários se deu pelo fato de serem instituições públicas que atendem às mulheres por motivo de nascimento. Os locais do estudo foram nominados de maternidade A, B e C, sendo uma maternidade-escola e as outras duas conveniadas com instituições de ensino para os cursos de graduação em Enfermagem e Medicina, e residência médica em Ginecologia e Obstetrícia. Todas as maternidades estavam pactuadas com as ações da Rede Cegonha e disponibilizavam orientações escritas para os acompanhantes. 
5 | Sabino MC, Costa R, Brüggemann OM, Junges CF, Gomes IEM, Velho MB

O macroprojeto desta pesquisa entrevistou 1.147 acompanhantes que permaneceram com a mulher durante o trabalho de parto e parto ou cesariana nessas maternidades. Para o recorte desta análise foi considerado apenas o número de acompanhantes que estiveram junto ao recém-nascido no momento dos cuidados imediatos, ao final participaram 1.075 acompanhantes.

Para integrar-se à pesquisa, o participante precisava estar de acordo com os seguintes critérios de inclusão: ser acompanhante de escolha da mulher que teve parto vaginal ou cesariana, gestação única e estar presente durante o trabalho de parto e parto normal ou cesariana, ocorrido há mais de quatro horas na maternidade. Foram critérios de exclusão: ter acompanhado mulheres que não entraram em trabalho de parto e mulheres que foram a óbito ou cujo recém-nascido tenha ido a óbito. Para a composição da amostra final, foram abordadas 4.239 puérperas durante a internação, destas 295 não tiveram o acompanhante continuamente no trabalho de parto e parto ou cesariana, 2.541 dos elegíveis não estavam presentes no Alojamento Conjunto, 13 acompanhantes não compreendiam a língua portuguesa e 229, recusaram-se a participar.

Os participantes foram abordados no Alojamento Conjunto de cada maternidade e, quando atendiam aos critérios de inclusão, eram convidados a participar da pesquisa. Havendo consentimento, realizavam-se as entrevistas face a face em outra sala, de preferência fora do quarto em que a mulher estava internada, em ambiente que proporcionasse uma interferência mínima, para não influenciar nas respostas.

O cálculo amostral do macroprojeto foi realizado a partir do número de nascimentos nessas maternidades no ano anterior à execução do projeto, tendo em vista a permanência de um acompanhante durante o trabalho de parto, parto ou cesariana. Considerando-se 3.508 nascimentos na maternidade A; 3.759, na maternidade B; e, 1.525 nascimentos na maternidade C; estabeleceu-se a prevalência presumida de 50\%, intervalo de confiança de $95 \%$, erro máximo de $5 \%$ e perda de $20 \%$, resultando em 346 entrevistados na maternidade A; 349 entrevistados na 
Ações realizadas pelo acompanhante durante os cuidados imediatos com o recém-nascido... 6

maternidade B; e, 307, na maternidade C. Assim, a amostra estimada de participantes foi de 1.002 acompanhantes e, conforme a disponibilidade de orçamento do macroprojeto, foi possível a realização de 1.147 entrevistas. Essa amostra foi estimada com o auxílio do SEstatNet', programa desenvolvido por docentes da Universidade Federal de Santa Catarina (UFSC). ${ }^{13}$

Os dados do macroprojeto foram coletados entre março de 2015 e maio de 2016, por estudantes de graduação em enfermagem. Na seleção dos entrevistadores tiveram prioridade aqueles que já tinham desenvolvido atividades teórico-práticas na área de saúde da mulher e do recém-nascido, e que conheciam as maternidades públicas do estudo. Os selecionados participaram de um treinamento que incluiu uma etapa teórica e outra prática.

Foram realizadas entrevistas face a face por meio da aplicação de um questionário digital pelos entrevistadores, para os quais se elaborou um software e, posteriormente, instalado em netbooks para uso exclusivo na pesquisa. O software foi criado a partir de um sistema informatizado que consistiu numa plataforma em que os dados foram armazenados digitalmente. As entrevistas ficaram salvas no formato de planilhas na extensão CSV, utilizando-se o Programa Microsoft Office Excel.

O questionário utilizado no macroprojeto se compunha por diferentes variáveis divididas em 10 seções. Nesse estudo foram selecionadas perguntas de algumas destas seções, sendo elas: Seção 1 - dados de identificação (idade, sexo, escolaridade, ocupação, estado civil, parentesco/vínculo com a mulher, número de filhos); Seção 2 - dados sobre a experiência de ser acompanhante (experiências anteriores e a atual); Seção 9 - dados sobre a sua participação na sala de cuidados com o recém-nascido.

Após o término da coleta de dados realizou-se a revisão dos questionários, com o intuito de sanar possíveis inconsistências ou erros e, após, exportaram-se os dados para o programa Stata versão 13.0. A análise descritiva apresentou a frequência absoluta e relativa, com seus respectivos intervalos de confiança de 95\%, das variáveis relacionadas às características 
7 | Sabino MC, Costa R, Brüggemann OM, Junges CF, Gomes IEM, Velho MB

sociodemográficas, à atuação dos profissionais de saúde na participação do acompanhante, e às ações dos acompanhantes nos cuidados imediatos com o recém-nascido.

As variáveis selecionadas para a análise foram: identificação do acompanhante, vínculo empregatício, escolaridade, vínculo com a parturiente, número de filhos, experiência anterior em ser acompanhante no trabalho de parto, parto e/ou cesariana, informação sobre a Lei do Acompanhante, participação em curso/palestra durante o pré-natal, encaminhamento do recém-nascido para a Unidade Neonatal, acompanhamento durante os cuidados prestados ao recém-nascido, permanência do acompanhante durante realização de procedimentos e exames no recém-nascido, ações realizadas pelo acompanhante durante os cuidados com o recém-nascido e sentimentos do acompanhante relacionados à experiência de acompanhar o recém-nascido na sala de cuidados.

O controle de qualidade foi realizado por ligações telefônicas, de forma aleatória, para 5\% dos acompanhantes entrevistados, ocasião em que se replicaram algumas perguntas do questionário. O macroprojeto no qual essa pesquisa estava inserida foi aprovado pelo Comitê de Ética e Pesquisa com Seres Humanos, sendo aprovado sob Certificado de Apresentação de Apreciação Ética número 25589614.3.0000.0121, no dia 25 de fevereiro de 2014. Todas as etapas da pesquisa respeitaram a Resolução número 466 de 2012, do Conselho Nacional de Saúde e os entrevistados autorizaram sua participação por meio da assinatura espontânea do Termo de Consentimento Livre e Esclarecido.

\section{Resultados}

Dos 1.075 acompanhantes que participaram da pesquisa, a maioria era do sexo masculino (77,9\%), estava na faixa etária dos $20-59$ anos $(94,3 \%)$ e possuía trabalho remunerado $(87,1 \%)$. O companheiro da mulher ou pai do recém-nascido representou quem mais esteve presente (77,6\%), a maioria dos acompanhantes desconhecia a Lei n. 11.108 (76,5\%) e não participou de 
Ações realizadas pelo acompanhante durante os cuidados imediatos com o recém-nascido... $\mid 8$

curso ou palestra sobre gestação/parto (91,7\%). As características sociodemográficas dos participantes podem ser observadas na Tabela 1.

Tabela 1 - Características sociodemográficas dos acompanhantes dos recém-nascidos nas três maternidades públicas da Grande Florianópolis. Florianópolis, SC. 2015-2016 (n=1.075)

\begin{tabular}{|c|c|c|c|}
\hline Variáveis & $\mathbf{N}$ & $\%$ & $I C^{*}(95 \%)$ \\
\hline \multicolumn{4}{|l|}{ Idade (anos) } \\
\hline$\leq 19$ & 46 & 4,3 & $3,0-5,5$ \\
\hline $20-59$ & 1014 & 94,3 & $92,9-95,7$ \\
\hline$\geq 60$ & 15 & 1,4 & $0,7-2,1$ \\
\hline \multicolumn{4}{|l|}{ Sexo } \\
\hline Masculino & 837 & 77,9 & $75,4-80,3$ \\
\hline Feminino & 238 & 22,1 & $19,7-24,6$ \\
\hline \multicolumn{4}{|l|}{ Ocupação } \\
\hline Trabalho remunerado & 936 & 87,1 & $85,1-89,1$ \\
\hline Trabalho sem remuneração & 80 & 7,4 & $5,9-9,0$ \\
\hline Desempregado & 39 & 3,6 & $2,5-4,7$ \\
\hline Aposentado/pensionista & 20 & 1,9 & $1,1-2,7$ \\
\hline \multicolumn{4}{|l|}{ Escolaridade } \\
\hline Sem escolaridade & 12 & 1,1 & $0,5-1,7$ \\
\hline Ensino Fundamental Incompleto & 277 & 25,8 & $23,1-28,4$ \\
\hline Ensino Fundamental Completo & 279 & 26,0 & $23,3-28,6$ \\
\hline Ensino Médio Completo & 403 & 37,5 & $34,6-40,4$ \\
\hline Ensino Superior Completo & 104 & 9,7 & $7,9-11,4$ \\
\hline \multicolumn{4}{|l|}{ Vínculo com a mulher } \\
\hline Companheiro ou pai do RN & 834 & 77,6 & $75,1-80,1$ \\
\hline Mãe & 128 & 12,0 & $10,0-13,9$ \\
\hline Mulher da rede social/familiar & 110 & 10,2 & $8,4-12,1$ \\
\hline Outros (pai, amigo, filho) & 3 & 0,3 & $0,0-0,6$ \\
\hline \multicolumn{4}{|c|}{ Conhecimento sobre a Lei do acompanhante } \\
\hline Sim & 253 & 23,5 & $21,0-26,1$ \\
\hline Não & 822 & 76,5 & $73,9-79,0$ \\
\hline \multicolumn{4}{|l|}{ Participação em curso e/ou palestra } \\
\hline $\operatorname{Sim}$ & 95 & 8,8 & $7,1-10,5$ \\
\hline Não & 980 & 91,2 & $89,5-92,9$ \\
\hline
\end{tabular}

$\mathrm{Na}$ Tabela 2 é possível observar que o enfermeiro foi quem mais incentivou o acompanhante a participar dos cuidados com o recém-nascido (46,1\%). A maioria dos 
profissionais de saúde forneceu informações sobre o atendimento prestado $(80,1 \%)$ e, destes, os acompanhantes apontaram o enfermeiro $(50,8 \%)$ com destaque na orientação sobre o seu papel.

Tabela 2 - Profissionais de saúde e a participação do acompanhante durante os cuidados imediatos com o recém-nascido. Florianópolis, SC. 2015-2016 (N=1.075)

\begin{tabular}{|c|c|c|c|}
\hline Variáveis & $\mathbf{N}$ & $\%$ & $\mathrm{IC}^{*}(95 \%)$ \\
\hline \multicolumn{4}{|l|}{ Profissional que convidou o acompanhante ${ }^{+}$} \\
\hline Nenhum profissional & 285 & 26,5 & $24,0-29,2$ \\
\hline O profissional não se identificou & 62 & 5,8 & $4,5-7,3$ \\
\hline Enfermeiro (a) & 496 & 46,1 & $43,2-49,1$ \\
\hline Médico (a) & 227 & 21,1 & $18,8-23,7$ \\
\hline Estudante de enfermagem & 2 & 0,2 & $0,1-0,7$ \\
\hline Estudante de medicina & 4 & 0,4 & $0,1-1,0$ \\
\hline Técnicos e auxiliares de enfermagem & 21 & 2,0 & $1,3-3,0$ \\
\hline Não lembra & 32 & 3,0 & $2,1-4,2$ \\
\hline \multicolumn{4}{|l|}{ Atitude dos profissionais de saúde ${ }^{t}$} \\
\hline Apresentaram-se para o acompanhante & 615 & 57,2 & $54,2-60,1$ \\
\hline Chamaram o acompanhante pelo nome & 279 & 26,0 & $23,4-28,7$ \\
\hline $\begin{array}{l}\text { Forneceram informações sobre a situação e o } \\
\text { atendimento prestado }\end{array}$ & 861 & 80,1 & $77,6-82,4$ \\
\hline $\begin{array}{l}\text { Perguntaram se o acompanhante tinha alguma } \\
\text { dúvida ou precisava de orientação }\end{array}$ & 518 & 48,2 & $45,2-51,2$ \\
\hline Perguntas realizadas pelo acompanhante ao & & & \\
\hline \multicolumn{4}{|l|}{ profissional de saúde } \\
\hline Não foram respondidas & 28 & 2,6 & $1,8-3,8$ \\
\hline Foram respondidas & 838 & 78,0 & $75,4-80,3$ \\
\hline Não realizou perguntas & 209 & 19,4 & $17,2-21,9$ \\
\hline \multicolumn{4}{|l|}{$\begin{array}{l}\text { Profissional que orientou sobre o papel do } \\
\text { acompanhante }^{+}\end{array}$} \\
\hline Nenhum profissional & 278 & 25,9 & $23,3-28,6$ \\
\hline Não se identificou & 85 & 7,9 & $6,4-9,7$ \\
\hline Enfermeiro (a) & 546 & 50,8 & $47,8-53,8$ \\
\hline Médico (a) & 294 & 27,4 & $24,8-30,1$ \\
\hline Estudante de enfermagem & 7 & 0,7 & $0,3-1,4$ \\
\hline Estudante de medicina & 17 & 1,6 & $1,0-2,5$ \\
\hline Técnicos e auxiliares de enfermagem & 53 & 4,9 & $3,8-6,4$ \\
\hline Não lembra & 3 & 0,3 & $0,1-0,9$ \\
\hline
\end{tabular}

${ }^{*}$ Intervalo de Confiança ${ }^{\dagger}$ Os participantes puderam escolher mais de uma opção 
Ações realizadas pelo acompanhante durante os cuidados imediatos com o recém-nascido... $\mid 10$

A Tabela 3 se refere às ações que o acompanhante realizou durante os cuidados imediatos com o recém-nascido e os sentimentos relacionados a essa experiência. Dentre os participantes, $94,8 \%$ conversaram com o recém-nascido, $93,0 \%$ o acariciaram, $81,4 \%$ o pegaram no colo, $67,6 \%$ ajudaram na primeira mamada e 20,4\% clampearam o cordão umbilical.

Em relação aos sentimentos, 99,3\% dos acompanhantes relataram que foi gratificante participar dos cuidados com o recém-nascido, 98,4\% referiram que a experiência os ajudou a ficarem tranquilos. E, ainda, $96,6 \%$ se sentiram seguros com o cuidado prestado ao recémnascido e $87,2 \%$ afirmaram que acompanhar o recém-nascido ajudou a tirar dúvidas sobre os cuidados com o bebê (Tabela 3 ).

Tabela 3 - Ações e sentimentos do acompanhante relacionados à experiência de acompanhar os cuidados imediatos prestados ao recém-nascido. Florianópolis, SC. 2015-2016 (N=1.075)

\begin{tabular}{|c|c|c|c|}
\hline Variáveis & $\mathbf{N}$ & $\%$ & $\mathrm{IC}^{*}(95 \%)$ \\
\hline \multicolumn{4}{|l|}{ Ações realizadas pelo acompanhante ${ }^{+}$} \\
\hline Cortou o cordão umbilical & 219 & 20,4 & $18,1-22,9$ \\
\hline Deu o primeiro banho & 85 & 7,9 & $6,4-9,7$ \\
\hline Acalmou o RN quando estava chorando & 842 & 78,3 & $75,8-80,7$ \\
\hline Fez carinho & 1.000 & 93,0 & $91,3-94,4$ \\
\hline Colocou a fralda & 287 & 26,7 & $24,1-29,4$ \\
\hline Ajudou a pesar & 459 & 42,7 & $39,8-45,7$ \\
\hline Pegou o RN no colo & 875 & 81,4 & $79,0-83,6$ \\
\hline Conversou com o RN & 1.019 & 94,8 & $93,3-96,0$ \\
\hline Levou o RN para a mulher após os cuidados & 628 & 58,4 & $55,4-61,3$ \\
\hline Ajudou na primeira mamada & 727 & 67,6 & $64,8-70,4$ \\
\hline Não realizou atividades relacionadas ao $\mathrm{RN}$ & 19 & 1,8 & $1,1-2,8$ \\
\hline Realizou outras atividades ${ }^{\#}$ & 61 & 5,7 & $4,4-7,2$ \\
\hline \multicolumn{4}{|l|}{ Sentimentos do acompanhante ao acompanhar o $\mathbf{R N}^{+}$} \\
\hline Ajudou a ficar tranquilo & 1.058 & 98,4 & $97,5-99,0$ \\
\hline Ajudou a tirar dúvidas sobre os cuidados com o RN & 937 & 87,2 & $85,0-89,0$ \\
\hline Deixou ansioso/nervoso & 491 & 45,7 & $42,7-48,7$ \\
\hline Ficou seguro com o cuidado prestado & 1.038 & 96,6 & $95,3-97,5$ \\
\hline Teve sentimento de obrigação em ser acompanhante & 493 & 45,9 & $42,9-48,9$ \\
\hline Foi gratificante & 1.064 & 99,3 & $98,5-99,6$ \\
\hline Achou o ambiente adequado para receber o acompanhante & 991 & 92,2 & $90,4-93,7$ \\
\hline Foi uma experiência que não quer viver novamente & 110 & 10,2 & $8,6-12,2$ \\
\hline
\end{tabular}

*Intervalo de Confiança ${ }^{\dagger}$ Os participantes puderam escolher mais de uma opção ${ }^{\#}$ Ajudou a secar, a colocar a roupa, a medir, a dormir, tirou foto, brincou, cantou, embalou, passeou, limpou o coto umbilical 
Os resultados apontam as ações desenvolvidas pelos acompanhantes e a forma como os profissionais de saúde interagem e estimulam para o exercício dessas, no cenário do nascimento. Descrevem os diferentes sentimentos vivenciados pela pessoa que está acompanhando a mulher e o recém-nascido, durante os cuidados imediatos.

\section{Discussão}

A predominância de acompanhantes do sexo masculino e a descrição do vínculo ser companheiro da mulher ou pai do recém-nascido coincidem com os achados de outras pesquisas realizadas no Brasil. A tendência pela presença do companheiro da mulher no cenário de parto favorece a formação de vínculo precoce do pai com o recém-nascido e aumenta a possibilidade de participação nos cuidados prestados no desenvolvimento infantil. ${ }^{8,14}$

A maioria dos participantes não conhecia a Lei do Acompanhante, apesar de 47,2\% terem o ensino médio completo ou maior nível de escolaridade. Também, a maioria, não participou de curso ou palestra sobre gestação ou parto. O desconhecimento acerca dos direitos no momento do nascimento apresenta repercussões e pode gerar submissão das mulheres e acompanhantes às instituições, bem como aos profissionais de saúde. ${ }^{15}$ Mesmo que a Lei obrigue os hospitais públicos ou conveniados ao Sistema Único de Saúde (SUS) a permitirem a presença de um acompanhante, estudos mostraram que ainda existem mulheres que permanecem sem acompanhamento no processo de parturição e nascimento. ${ }^{5,16}$ A realização de cursos, o repasse de informações, por meio das consultas de pré-natal com reforço desse direito até a chegada na maternidade e por todos os profissionais, pode ser uma estratégia para informar esta população..$^{1,4}$

O convite feito para o entrevistado permanecer junto ao recém-nascido foi realizado preponderantemente pelo enfermeiro, que também foi a categoria reconhecida pelo participante como o profissional que mais lhe forneceu orientações. O enfermeiro desempenha papel 
Ações realizadas pelo acompanhante durante os cuidados imediatos com o recém-nascido... $\mid 12$

importante no que tange às orientações passadas para esse acompanhante, as quais conferem segurança e auxiliam na aquisição de habilidades necessárias para a realização dos cuidados com o recém-nascido..$^{8,17}$

As maiores dificuldades para inserção do acompanhante, por parte da equipe de saúde no processo de nascimento, estiveram relacionadas à falta de preparo do mesmo para lidar com esse momento, situação que eventualmente pode interferir de forma negativa no serviço da equipe. ${ }^{18}$ Cabe ao profissional de saúde a missão de orientar e direcionar as ações do acompanhante, estimulá-lo a estar presente desde o pré-natal para compreender com maior clareza seu papel durante todo o processo. ${ }^{19} \mathrm{O}$ resultado desta pesquisa mostrou a disponibilidade dos profissionais de saúde na inserção do acompanhante, ao responderam a maioria das perguntas realizadas por ele.

No que se refere à atuação dos acompanhantes nos cuidados imediatos junto ao recémnascido, evidenciou-se que as ações mais frequentes foram conversar com o recém-nascido, fazer carinho nele e acalmá-lo. Ressalta-se que ações de caráter sentimental e afetivo influenciam na consolidação das relações familiares e estreitamento de vínculos. ${ }^{20}$ Neste estudo, percebeu-se que grande parte dos acompanhantes tiveram o primeiro contato físico com o recém-nascido durante a realização dos cuidados imediatos, sendo relevante a este aspecto o ato de pegar o recém-nascido no colo, ajudar a pesá-lo e conduzi-lo até a mulher após os cuidados.

Outro dado que se destacou, esteve relacionado ao auxílio prestado pelo acompanhante ao recém-nascido e à mãe durante a primeira mamada, observou-se que mais da metade dos entrevistados participou ativamente deste momento. O início do aleitamento materno é um desafio tanto para a mãe quanto para o acompanhante, sendo necessário que sejam realizadas ações organizadas e planejadas por parte dos profissionais de saúde para que se obtenha sucesso. ${ }^{21}$ Nos resultados deste estudo, o acompanhante simbolizou uma ligação entre a família e o profissional de saúde, ou seja, foi o intermediário das necessidades familiares enquanto a 
13 | Sabino MC, Costa R, Brüggemann OM, Junges CF, Gomes IEM, Velho MB

mulher não conseguia acompanhar os cuidados com o recém-nascido, a exemplo da impossibilidade das mulheres saírem do leito, quando submetidas à cesariana.

O acompanhante deve ser estimulado e inserido neste processo a fim de favorecer o aleitamento materno, além de realizar outras ações como clampear o cordão umbilical, colocar a fralda ou dar o primeiro banho, ${ }^{1}$ atividades efetuadas por menos de um terço dos participantes da pesquisa. Em contrapartida, notou-se que poucos entrevistados relataram que não fizeram nenhuma atuação junto ao recém-nascido.

Os sentimentos do acompanhante também foram evidenciados e destaca-se a afirmação da grande maioria, de ter sido uma experiência gratificante, fato que ajudou o acompanhante a ficar tranquilo e a se sentir seguro com o cuidado prestado. Estudos afirmam que participar deste período é importante para o acompanhante, momento que representa um marco em sua vida, ao conhecer o novo membro da família, além de reafirmar a consolidação das relações familiares e o estreitamento de vínculos. ${ }^{7,20-22}$

Alguns participantes $(45,7 \%)$ declararam que presenciar este momento os deixaram ansiosos, nervosos ou que tiveram o sentimento de obrigação em ser acompanhante. Em número ainda menor (10,2\%), afirmaram que não desejavam vivenciar novamente esta experiência. Isto demonstra que todos descreveram algum tipo de sentimento envolvido com a sua participação, no momento dos cuidados imediatos prestados ao recém-nascido. São sentimentos e afirmações que precisam de atenção e justifica-se novamente, neste resultado, a importância da preparação prévia dos acompanhantes, seja por meio da participação em grupos, cursos ou nas consultas durante o acompanhamento pré-natal.

Ainda, fez parte da percepção da maioria dos entrevistados que o ambiente foi adequado para receber o acompanhante $(92,2 \%)$. Entende-se como ambiente adequado um local aconchegante, com rotinas flexíveis, no qual a mulher e família consigam se expressar livremente. ${ }^{1}$ Em porcentagem um pouco menor, mas ainda expressiva $(87,2 \%)$, relataram que foi 
Ações realizadas pelo acompanhante durante os cuidados imediatos com o recém-nascido... $\mid 14$

possível sanar as dúvidas sobre os cuidados com o recém-nascido. Este dado permite inferir que o acompanhante, quando estimulado e preparado para participar ativamente do processo parturitivo, realiza atividades que auxiliam a mulher, ao passo que se sentem acolhidos para realizar os cuidados, mesmo que apresentem dúvidas e inseguranças.

Cabe salientar que a presença e participação do acompanhante nos cuidados imediatos ao recém-nascido possibilita uma melhor adaptação do mesmo à vida extrauterina. Também contribui para o aprendizado sobre as necessidades do bebê e os cuidados que devem ser prestados a ele, como realizar a higiene, trocar fraldas, entre outros, ${ }^{17}$ facilitando a adaptação e tornando o dia a dia com o novo membro familiar mais tranquilo.

Como limitações identificadas neste estudo destaca-se o fato de que, quando os participantes realizaram cursos e palestras, os conteúdos não foram avaliados, tampouco foram avaliadas as ações de intermédio do acompanhante para o atendimento das solicitações da mulher, relacionadas aos cuidados com o recém-nascido. Apesar disso, acredita-se que o estudo é inovador e apresenta dados acerca das ações realizadas pelo acompanhante durante os cuidados com o recém-nascido, pouco avaliadas em estudos anteriores.

\section{Conclusão}

A participação do acompanhante nos cuidados com o recém-nascido demonstra ações de caráter sentimental, afetivo e de cuidado e, ainda, facilita o estabelecimento dos vínculos familiares. Conhecer as ações realizadas pelos acompanhantes agrega valor às suas presenças, ao passo que ratifica a necessidade de ser e estar no cenário de parto e nascimento, pois não se configuram apenas como espectadores. Destaca-se que a maioria dos entrevistados realizou algum tipo de ação voltada ao recém-nascido e, identificar as mesmas e a sua relevância no processo de nascimento é importante para a consolidação da figura do acompanhante como elemento integrado ao processo parturitivo. 
Os benefícios relacionados à presença do acompanhante junto ao recém-nascido e à mulher justificam a relevância dos estudos que tenham como objeto de pesquisa os diferentes aspectos relacionados ao próprio acompanhante. Desta forma, acredita-se que esta pesquisa colaborou com a descrição e análise das ações por ele realizadas durante os cuidados imediatos com o recém-nascido, a importância da sua presença e novas formas de atuação neste cenário. Em vista disso, o estudo também oferece subsídios para a prática assistencial uma vez que seus resultados demonstram potencialidades de participação do acompanhante, uma colaboração que pode auxiliar para uma experiência positiva de parto e nascimento, com maior significado para a mulher e à família.

\section{Referências}

1. Ministério da Saúde (BR). Diretriz nacional de assistência ao parto normal: relatório de recomendação [Internet]. Brasília (DF): Ministério da Saúde; 2016 [acesso em 2016 jul 24]. Disponível em: http://conitec.gov.br/images/Consultas/2016/Relatorio_Diretriz-PartoNormal_CP.pdf

2. World Health Organization (WHO). WHO recommendations: intrapartum care for a positive childbirth experience [Internet]. Geneva: World Health Organization; 2018 [cited 2018 Jul 24]. Available from: https://www.who.int/publications-detail/9789241550215

3. Tunçalp Ö, Were WM, MacLennan C, Oladapo OT, Gülmezoglu AM, Bahl R, et al. Quality of care for pregnant women and newborns-the WHO vision. BJOG. 2015;122(8):1045-9. doi: https://doi.org/10.1111/1471-0528.13451

4. BRASIL. Lei no 11.108, de 07 de abril de 2005. Altera a Lei no 8.080, de 19 de setembro de 1990, para garantir às parturientes o direito à presença de acompanhante durante o trabalho de parto, parto e pósparto imediato, no âmbito do Sistema Único de Saúde SUS. Diário Oficial da União: seção 1, Brasília, DF, ano 142, n. 67, p. 1, 08 abr. 2005.

5. Monguilhott JJC, Brüggemann OM, Freitas PF, d'Orsi E. Nascer no Brasil: the presence of a companion favors the use of best practices in delivery care in the South region of Brazil. Rev Saúde Pública. 2018;52:1. doi: https://doi.org/10.11606/s1518-8787.2018052006258

6. Carvalho CFS, Carvalho IS, Brito RS, Vitor AF, Lira ALBC. O companheiro como acompanhante no processo de parturição. Rev Rene. 2015;16(4):613-21. doi: https://doi.org/10.15253/21756783.2015000400019 
Ações realizadas pelo acompanhante durante os cuidados imediatos com o recém-nascido... $\mid 16$

7. Francisco BS, Souza BS, Vitório ML, Zampieri MFM, Gregório VRP. Fathers' perceptions about their experiences as birth companions. REME Rev Min Enferm. 2015;19(3):576-83. doi: https://doi.org/10.5935/1415-2762.20150044

8. Brüggemann OM, Koettker JG, Velho MB, Monguilhott JJC, Monticelli M. Satisfação dos acompanhantes com a experiência de apoiar a parturiente em um hospital universitário. Texto Contexto Enferm. 2015;24(3):686-96. doi: https://doi.org/10.1590/0104-07072015004220014

9. Dodou HD, Sousa AAS, Barbosa EMG, Rodrigues DP. Sala de parto: condições de trabalho e humanização da assistência. Cad Saúde Colet (Rio J). 2017;25(3):332-8. doi: https://doi.org/10.1590/1414$462 X 201700030082$

10. Van der Gucht N, Lewis K. Women's experiences of coping with pain during childbirth: a critical review of qualitative research. Midwifery. 2015;31(3):349-58. doi: https://doi.org/10.1016/j.midw.2014.12.005

11. Bohren MA, Hofmeyr GJ, Sakala C, Fukuzawa RK, Cuthbert A. Continuous support for women during childbirth. Cochrane Libr. 2017. 7:CD003766. doi: https://doi.org/10.1002/14651858.CD003766.pub6

12. Ministério da Saúde (BR). Guia do pré-natal do parceiro para profissionais de saúde [Internet]. Brasília (DF): Ministério da Saúde; 2016 [acesso em 2020 out 27]. Disponível em: https://portalarquivos.saude.gov.br/images/pdf/2016/agosto/11/guia_PreNatal.pdf

13. Nassar SM, Wronscki VR. SEstatNet - Sistema Especialista para o Ensino de Estatística na Web [Internet]. 2015 [acesso em 2016 jul 24]. Disponível em: http://sestatnet.ufsc.br

14. Anjos AM, Gouveia HG. Presença do acompanhante durante o processo de parturição e nascimento: análise da prática. Rev Enferm UERJ. 2019;27:e38686. doi: https://doi.org/10.12957/reuerj.2019.38686

15. Rodrigues DP, Alves VH, Penna LHG, Pereira AV, Branco MBLR, Souza RMP. O descumprimento da lei do acompanhante como agravo à saúde obstétrica. Texto Contexto Enferm. 2017;26(3):e5570015. doi: https://doi.org/10.1590/0104-07072017005570015

16. Nascimento SL, Pires VMMM, Santos NA, Machado JC, Meira LS, Palmarella VPR. Conhecimentos e experiências de violência obstétrica em mulheres que vivenciaram a experiência do parto. Revenf [Internet]. 2019 [acesso em 2020 nov 11];(37):66-79. Disponível em: https://www.scielo.sa.cr/scielo.php?script=sci_arttext\&pid=S1409-45682019000200066

17. Silva CS, Carneiro MNF. Pais pela primeira vez: aquisição de competências parentais. Acta Paul Enferm. 2018;31(4):366-73. doi: https://doi.org/10.1590/1982-0194201800052

18. Bohren MA, Berger BO, Munthe-Kaas H, Tunçalp Ö. Perceptions and experiences of labour companionship: a qualitative evidence synthesis. Cochrane Libr. 2019;3:CD012449. doi: https://doi.org/10.1002/14651858.CD012449.pub2

19. Holanda SM, Castro RCMB, Aquin PS, Pinheiro AKB, Lopes LG, Martins ES. Influência da 
participação do companheiro no pré-natal: satisfação de primíparas quanto ao apoio no parto. Texto Contexto Enferm. 2018;27(2):e3800016. doi: https://doi.org/10.1590/0104-070720180003800016

20. Gomes IEM, Padoin SMM, Langendorf TF, Paula CC, Gomes CA, Ribeiro AC. Benefícios da presença do acompanhante no processo de parto e nascimento: revisão integrativa. Rev Enferm UFSM. 2019;9(e61):1-18. doi: https://doi.org/10.5902/2179769234170

21. Gomes ALM, Rocha CR, Henrique DM, Santos MA, Silva LR. Conhecimentos de familiares sobre os cuidados com recém-nascidos. Rev Rene. 2015;16(2):258-65. doi: https://doi.org/10.15253/21756783.2015000200016

22. Souza SRRK, Gualda DMR. A experiência da mulher e de seu acompanhante no parto em uma maternidade pública. Texto Contexto Enferm. 2016;25(1):e4080014. doi: https://doi.org/10.1590/01040707201600004080014

Editor Científico: Tânia Solange Bosi de Souza Magnago

Editor Associado: Graciela Dutra Sehnem

Fomento / Agradecimento: Conselho Nacional de Desenvolvimento Científico e Tecnológico (CNPq) - Edital Universal 14/2013.

\section{Autor correspondente}

Manuela Beatriz Velho

E-mail: manuela.velho@ufsc.br

Campus Universitário - Trindade

88.040-900 - Florianópolis - SC - Brasil

BLOCO I (CEPETEC) - Centro de Ciências da Saúde - Sala 420

\section{Contribuições de Autoria}

\section{1 - Mayara Carminatti Sabino}

Concepção ou desenho do estudo/pesquisa, análise e/ou interpretação dos dados, revisão final com participação crítica e intelectual no manuscrito.

\section{2 - Roberta Costa}

Concepção ou desenho do estudo/pesquisa, análise e/ou interpretação dos dados, revisão final com participação crítica e intelectual no manuscrito.

\section{3 - Manuela Beatriz Velho}

Concepção ou desenho do estudo/pesquisa, revisão final com participação crítica e intelectual no manuscrito. 
Ações realizadas pelo acompanhante durante os cuidados imediatos com o recém-nascido... | 18

\section{4 - Odaléa Maria Brüggemann}

Concepção ou desenho do estudo/pesquisa, revisão final com participação crítica e intelectual no manuscrito.

\section{5 - Carolina Frescura Junges}

Concepção ou desenho do estudo/pesquisa, revisão final com participação crítica e intelectual no manuscrito.

\section{6 - Iris Elizabete Messa Gomes}

Concepção ou desenho do estudo/pesquisa, revisão final com participação crítica e intelectual no manuscrito.

\section{Como citar este artigo}

Sabino MC, Costa R, Velho MB, Brüggemann OM, Junges CF, Gomes IEM. Ações realizadas pelo acompanhante durante os cuidados imediatos com o recém-nascido em maternidades públicas. Rev. Enferm. UFSM. 2021 [Acesso em: Anos Mês Dia]; vol.11 e26: 1-18. DOI: https://doi.org/10.5902/2179769246916 